\title{
Molecular-Weight Dependence of the Formation of Extended-Chain Crystals in Polyethylene under High Pressure
}

\author{
Shuichi SAwada, Ken Kato, and Takuhei NosE \\ Department of Polymer Chemistry, Tokyo Institute of Technology, \\ Ookayama, Meguro-ku, Tokyo 152, Japan.
}

(Received December 25, 1978)

\begin{abstract}
The dependence of the formation of extended-chain crystals under high pressure on molecular weight was investigated in a quantitative way for seven kinds of fractionated polyethylenes with different molecular weights ranging from 4300 to 410000 . The extent to which extended-chain crystals were produced in the cooling crystallization increased with increasing pressure. It was shown that the pressure at which the changeover from folded-chain to extendedchain crystallization took place was inversely proportional to the molecular weight in the range of the molecular weight studied. To explain the molecular-weight dependence, a model of a bundle-like nucleus formation is proposed as a crystallization mechanism of extended-chain crystals. It is shown that longer molecules are kinetically favorable for producing extended-chain crystals.

KEY WORDS Polyethylene / High Pressure / Extended-Chain Crystals /

Transition Pressure / Molecular-Weight Dependence / Bundle-Nucleus / Longitudinal Growth / Stochastic Process /
\end{abstract}

In the case of atmospheric-pressure crystallization, the production of extended-chain crystals became difficult with increasing molecular weight. ${ }^{1}$ But in the case of high-pressure crystallization, it has been shown qualitatively that the formation of extended-chain crystals is much easier for samples of higher molecular weight. ${ }^{2-4}$ Thus we have proposed that this discrepancy stems from the difference in the mechanism of the formation of extended-chain crystals at atmospheric pressure and high pressures. ${ }^{5}$ The case of atmospheric-pressure crystallization may be explained in consideration of the fact that the formation of extended-chain crystals for high molecular weight is difficult owing to the high free energy created on formation of a large critical nucleus.

As for the formation of extended-chain crystals under high pressure, another mechanism should be taken into account to explain the somewhat paradoxical problem of the molecular-weight dependence. Up to the present, however, there has been little discussion about this matter. Bassett et al. have pointed out that the location of the triple point in the phase diagram, correlated to the formation of extended-chain crystals, was dependent on molecular weight; a higher molecular weight sample gave a triple point at a lower pressure. ${ }^{6}$ Hence they propose that extended-chain crystals are easily produced for longer molecules. However, it was not clarified as to why the position of the triple point changed with molecular weight.

In this paper, we investigate this molecular-weight dependence problem in a quantitative way and attempt to consider the problem from a kinetic point of view.

\section{EXPERIMENTAL}

The samples employed in this study were fractionated polyethylenes $\left(M_{w} / M_{n}=1.2-1.3\right)$ with the viscosity-average molecular weights $M_{v}=4300$, $10000,15500,17300,30000,150000$, and 410000 .

A differential thermal analysis (DTA) cell used in this high-pressure experiment was reported

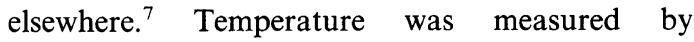
chromel-alumel thermocouples and pressure was measured by using the change in the electric conductivity of manganin wire within an accuracy of $\pm 10 \mathrm{~kg} \mathrm{~cm}^{-2}$.

In each run, polyethylene crystallized at a constant cooling rate of $1^{\circ} \mathrm{C} / \mathrm{min}$ at atmopsheric pressure was used as the starting material. To investigate the melting and crystallization behavior under pressure, 
first, the pressure was raised to the desired value and kept constant at room temperature. Then, the temperature was raised at a heating rate of $2^{\circ} \mathrm{C} / \mathrm{min}$, and the melting temperature under pressure was determined with the DTA technique. After the complete melting of the sampe, the temperature was lowered at a rate of $1.5^{\circ} \mathrm{C} / \mathrm{min}$, and the crystallization temperature under pressure was determined. Pressure was released after assuring that the temperature was brought near to room temperature. The melting temperature of the materials crystallized under pressure was measured at atmospheric pressure.

For each run, the temperature corresponding to the peak of endothermic trace was taken as the melting temperature, and the crystallization temperature was determined as the point at which the first deviation from the base line could be observed. ${ }^{8}$

\section{RESULTS}

The dependences of the melting and crystallization temperatures on pressure for $M_{v}=150000$ are shown in Figure 1 with th pressure dependence of the melting temperature of extended-chain crystals produced under high pressure. The crystallization temperature versus the pressure relation was not described by a single curve and divided into two parts at a high pressure depending on molecular weight. In the region of the discontinuity of the crystallization temperature curve, the DTA traces had double

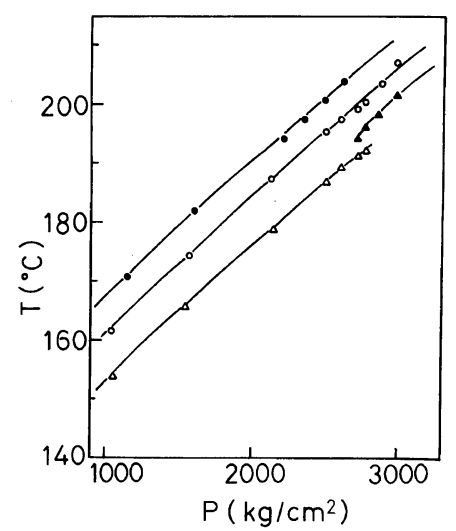

Figure 1. The melting and crystallization temperatures $v s$. pressure for $M_{v}=150000$. (O) denotes the melting temperature of extended-chain crystals, $(O)$ that of folded-chain crystals; $(\boldsymbol{\Delta})$ the crystallization temperature of extended-chain crystals, and $(\triangle)$ that of folded-chain crystals. peaks, as some authors have reported. ${ }^{6,9}$

Corresponding to these facts, for the materials crystallized at various pressures, the transition from a low to a high melting temperature at atmospheric pressure was observed around a certain crystallization pressure, $P_{1}$, which is called here the transition pressure, and depends on the molecular weight. As an example, Figure 2 shows the plot of the melting

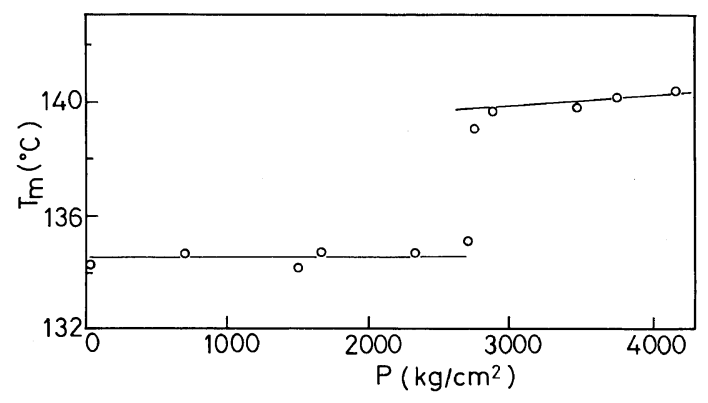

Figure 2. The melting temperatures at atmospheric pressure for the materials crystallized at various pressures are plotted against the pressure under crystallization for $M_{v}=30000$.
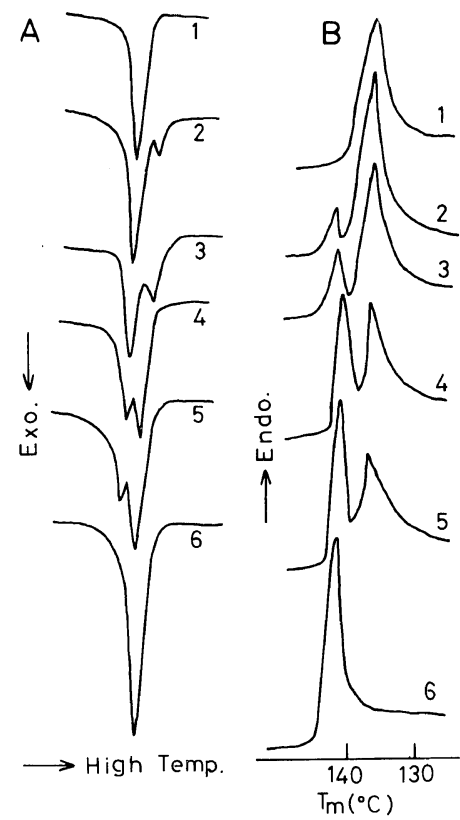

Figure 3. The DTA traces for $M_{v}=17300$. (A) denotes the exothermic traces at various pressures and (B) denotes the endothermic traces at atmospheric pressure of the materials crystallized under pressure. Pressure at (1) $2510 \mathrm{~kg} \mathrm{~cm}^{-2}$, (2) $2800 \mathrm{~kg} \mathrm{~cm}^{-2}$, (3) $2840 \mathrm{~kg} \mathrm{~cm}^{-2}$, (4) $2930 \mathrm{~kg} \mathrm{~cm}^{-2}$, (5) $2970 \mathrm{~kg} \mathrm{~cm}^{-2}$, and (6) $3320 \mathrm{~kg} \mathrm{~cm}^{-2}$. 
temperatures at atmospheric pressure for the materials crystallized under each pressure against the pressure during crystallization for a molecular weight of 30000 .

In order to investigate the crystallization behavior in the transitio region where double peaks in DTA traces appeared, detailed studies of the crystallization near the transition pressure were carried out. The exothermic traces under pressure and the endothermic traces at atmospheric pressure of pressure-crystallized materials are coupled in Figure 3 for molecular weight 17300 , as an example. The exotermic traces were shifted along the temperature scale so that each trace came to the same position, in order to show the change in the shape of the trace with pressure. The proportion of extended-chain crystals produced under pressure crystallization, estimated from the area of the endothermic peak, is tabulated against the pressure under crystallization in Table I.

The transition pressure $P_{\mathrm{t}}$ was plotted against the inverse of the molecular weight in Figure 4. In the

Table I. Proportion of extended-chain crystals

\begin{tabular}{cc}
\hline Pressure & Proportion \\
\cline { 2 - 2 } $\mathrm{kg} \mathrm{cm}^{-2}$ & \\
\hline 2740 & 0.05 \\
2800 & 0.12 \\
2840 & 0.22 \\
2930 & 0.43 \\
2970 & 0.63 \\
3050 & 0.77 \\
3150 & 0.90 \\
3310 & 1.00 \\
\hline
\end{tabular}

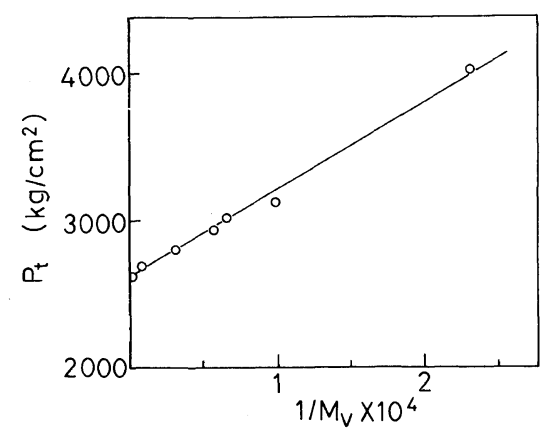

Figure 4. Plot of the transition pressure $P_{\mathrm{t}}$ against the inverse of molecular weight. range of the molecular weight studied, the transition pressure was inversely proportional to the molecular weight.

\section{DISCUSSION}

It is considered that the lamellar thickness of folded-chain crystls produced at low pressures varies little with pressure during crystallization. ${ }^{7}$ This seems to reflect the almost constant value of the melting temperatures at low ressures as shown in Figure 2. In Figure 2, it may be supposed that lowpressure-crystallized materials correspond to foldedchain crystals and that high-pressure-crystallized materials to extended-chain crystals. Consequently, we identify two crystallizationtemperature cruves in Figure 1: the high crystallization temperature curve is for extended-chain crystallization, and the low one for chain-folded crystallization.

In Figure 3, it is easily seen that the extent of extended-chain crystals steadily increased with pressure applied. This fact shows that pressure contributes to the increase in the rate of extended-chain crystallization, and that the rate of extended-chain crystallization overcomes that of folded-chain crstallization above a pressure $P_{1}$ in the cooling crystallization. This result is the same as in the case of isothermal crystallization in which pressure plays an important role in forming extended-chain crystals. ${ }^{7.10}$

Figure 4 shows that the transition pressure $P_{\mathrm{t}}$ has a linear relationship to the inverse molecular weight. It is worthy to note that there should be no transtion pressure for low-molecular-weight polyethylenes giving rise to fully extended-chain crystals in the cooling crystallization atatmospheric pressure. In this case, forming extended-chain crystals has nothing to do with pressure. It should be mentioned that the transition pressures in Figure 4 hold for the cooling rate conducted in this experiment. $P_{\mathrm{t}}$ depends on the cooling rate for crystallization; for a slower cooling rate, it was reported that the formation of extended-chain crystals is possible at a lower pressure, ${ }^{9}$ that is, $P_{1}$ decreases.

It has been shown that extended-chain and foldedchain crystallization are mutually independent processes, ${ }^{4,9,11,12}$ and it is possible that extended-chain crystallization takes place prior to folded-chain crystallization. ${ }^{6,7,9}$ Therefore, as an extended-chain crystallization mechanism, it is appropriate to con- 
sider crystallization with nonfoldd-chain structures. In the nucleation of nonfolded-chain crystallization, there are two possibilities: one is the attachment of whole molecule to the crystal substrate and the other is the attachment of part of a molecule to th substrate. For long molecules, the former situation is unfavorable because of the high free energy created on formin a thick nucleus. It would be reasonable to consider that each molecule is partially incorporated into the crystal substrate. From these considerations, we assume that a bunde-like nucleus formation is the most likely as a nonfolded-chain nucleation under high pressure.

Some authors have pointed out that it is difficult to form a bundle-like nucleus, since the value of the excess free energy of nucleus surface normal to the chain direction, $\sigma_{\mathrm{e}}$, is high for a bundlenucleus. ${ }^{13,14}$ This difficulty may be overcome by considering tat the value of the excess free energy of a bundle-like nucleus surface, $\sigma_{\mathrm{eb}}$, decreases considerably with increasing pressue.

It is known that both folded-chain and extendedchain crystallization rates increase with increasing pressure. $^{7,10,15}$ Since the crystallizatio rate is sensitive to the value of $\sigma_{\mathrm{e}}$, it may be concluded that the value of $\sigma_{\mathrm{e}}$ for both cases is reduced. From the data of the isothermal crystallization rates, it was estimated that the value of the excess free energy of fold surface, $\sigma_{\mathrm{ef}}$, a pressure $P=3000$ was a half of that at atmospheric pressure. ${ }^{7}$ But, it is natural that there should be a lower limit on the value of $\sigma_{\text {ef }}$ corresponding to the lowest energy to form the fold surface. On the other hand, there seems to be no reaso to place a limit on $\sigma_{\mathrm{e}}$ for the bundle-like nucleus. To illustrate this stituation, the dependence of $\sigma_{\mathrm{e}}$ on pressure is schematically shown in Figure 5. It is considered that bundle-like crystallization becomes favorable compared with folded-chain crystallization owing to the considerable depression of the value of $\sigma_{\mathrm{e}}$ as pressure is increased.

Contrary to the case of foded-chain crystals, the crystal growth along the chain direction is possible for bundle-like crystals. Hence, we introduce the factor of longitudinal growth of the chain. It is considered that this longitudinal growth, which corresponds to the lamellar thickening in the case of folded-chain crystals, assures the thickness of the extended-chain crystals observed at atmospheric pressure.

We now estimate the surface nulceation rate for a

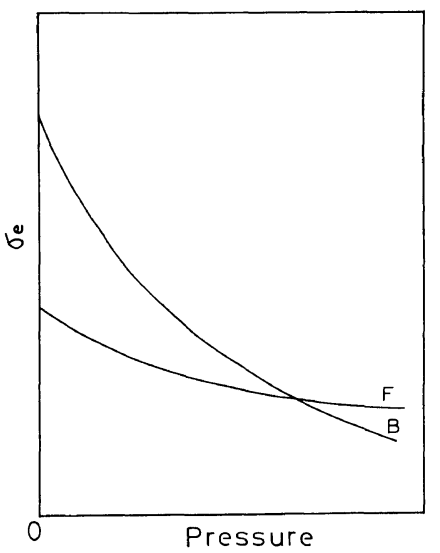

Figure 5. Schematic diagram of the dependence of the value of $\sigma_{\mathrm{e}}$ on pressure. (F) denotes folded-chain nucleus and (B) denotes bundle-like nucleus.

bundle-like nucleus, by using the method of the stochastic processes. This mehod has been developed for folded-chain crystals. ${ }^{16-18}$

We denote $\alpha_{v-1}$ and $\beta_{v}$ as the forward and backward transition rates between the states $v-1$ and $v$, respectively. In general, the net transition rate $J_{v}$ between the states $v-1$ and $v$ is

$$
J_{v}=\alpha_{v-1} N_{v-1}-\beta_{v} N_{v}
$$

where $N_{v-1}$ and $N_{v}$ are the occupation numbers at the states $v-1$ and $v$, respectively. The occupation number $N_{v}$ is time-dependent and given by

$$
\frac{\mathrm{d} N_{v}}{\mathrm{~d} t}=J_{v}-J_{v+1}
$$

$\mathrm{d} N_{v} / \mathrm{d} t=0$ under steady-state conditions.

In this model, it is denoted that, except for the first ste which has transition rate pairs $\alpha_{0}$ and $\beta_{1}$, the following steps have the same transition rate pairs $\alpha_{1}$ and $\beta_{2}$. The net transition rates are

$$
\begin{gathered}
J_{1}=\alpha_{0} N_{0}-\beta_{1} N_{1} \\
J_{2}=\alpha_{1} N_{1}-\beta_{2} N_{2} \\
\quad \cdots \cdots \cdots \cdots \\
J_{v}=\alpha_{1} N_{v-1}-\beta_{2} N_{v}
\end{gathered}
$$

For simplicity of treatment, steady-state conditions were imposed. Therefore, the net transition rates are all equal from eq 2 and can be described by $J$. Each equation in eq 3 except the first is multiplied by $\left(\beta_{1} / \alpha_{1}\right)\left(\beta_{2} / \alpha_{1}\right)^{i-2}, i=2,3, \cdots, v$, and all equations are added up to obtain 


$$
J=A_{0} N_{0}-B_{1} N_{v}
$$

where

$$
\begin{aligned}
& A_{0}=\frac{\alpha_{0}}{1+\left(\beta_{1} / \alpha_{1}\right) \sum_{k=1}^{v-1}\left(\beta_{2} / \alpha_{1}\right)^{k-1}} \\
& B_{1}=\frac{\beta_{1}\left(\beta_{2} / \alpha_{1}\right)^{v-1}}{1+\left(\beta_{1} / \alpha_{1}\right) \sum_{k=1}^{v-1}\left(\beta_{2} / \alpha_{1}\right)^{k-1}}
\end{aligned}
$$

The condition $\beta_{2} / \alpha_{1}<1$ has to be satisfied in order to assure nucleation growth, and if $v$ is large we obtain the steady-state solution

$$
J=\frac{\alpha_{0}\left(\alpha_{1}-\beta_{2}\right) N_{0}}{\alpha_{1}+\beta_{1}-\beta_{2}}
$$

It should be mentioned that the crystal substrate required for the surface nucleation growth was implicitly assumed to be present.

According to Flory, ${ }^{19}$ Mandelkern, ${ }^{20}$ and Price, ${ }^{21}$ the free-energy change in formation, such as for a bundle-like nucleus displayed in Figure 6, can be expressed as

$$
\begin{aligned}
\Delta F= & 2 b_{0} l \sigma+2 m a_{0} b_{0} \sigma_{\mathrm{eb}}-m a_{0} b_{0} l \Delta g \\
& +k T\left[\frac{m l}{L c_{0}}-m \ln \frac{L-n+1}{L}\right]
\end{aligned}
$$

where $a_{0}, b_{0}$, and $c_{0}$ are the dimensions of the molecules comprising the bundle-like nuceus, $m$, the number of molecules in the nuclus, $n$, the number of repeat units in a crystalline sequence, $l=n c c_{0}$ the thickness of the bundle-like nucleus, $L$, the contour length of a molecule in number of repeat units, $\Delta g$, the bulk free energy of fusion, $\sigma$, the excess free energy of the lateral surface, $T$, the crystallization temperature, and $k$, Boltzmann's constant. The bulk free energy of fusion $\Delta g$ is given by $\Delta h \Delta T / T_{\mathrm{m}}{ }^{0}$, where $\Delta h$ is the heat of fusion, $\Delta T$, the supercooling, and $T_{\mathrm{m}}{ }^{0}$, the equilibrium melting temperature. In eq 8 , the fourth term results from the increased volume available to the ends of the polymer chains on melting and the fifth term results mainly from the requirement that the ends of the molecules should stay out of the crystallites. Both terms are entropy terms giving the molecular-weight dependence of the

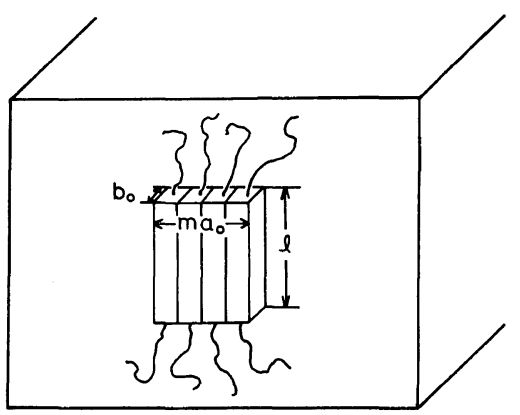

Figure 6. Model of monomolecular growth of bundlelike nucleus.

formation of bundle-like nucleus. Thus, the transition rates were chosen in the following way

$$
\begin{gathered}
\alpha_{0}=\frac{k T}{h} \exp \left[-\left\{2 b_{0} l \sigma+2 b_{0}^{2} \sigma_{\mathrm{eb}}\right.\right. \\
\left.\left.+k T\left(\frac{n}{L}-\ln \frac{L-n+1}{L}\right)-0.5 b_{0}^{2} l \Delta g\right\} / k T\right] \\
\alpha_{1}=\frac{k T}{h} \exp \left[-\left\{2 b_{0}^{2} \sigma_{\mathrm{eb}}\right.\right. \\
\left.\left.+k T\left(\frac{n}{L}-\ln \frac{L-n+1}{L}\right)-0.5 b_{0}^{2} l \Delta g\right\} / k T\right] \\
\beta_{1}=\beta_{2}=\frac{k T}{h} \exp \left(-0.5 b_{0}^{2} l \Delta g / k T\right)
\end{gathered}
$$

where $h$ is Planck's constant. We assume in this model that the free-energy gain $b_{0}{ }^{2} l \Delta g$ on attaching a stem of $l$ to the crystal substrate is equally shared in the forward and backward steps, because the mechanism of the incorpolation of molecules is not sufficiently clarified. Selection of the way for apportioning the free-energy gain will not affect the results appreciably. For simplicity, the case $a_{0}=b_{0}$ was considered.

In the case of nucleation-controlled crystal growth, which is probably justified in the case of crystallization at small supercoolings such as high-pressure crystallization, the growth rate $G$ and net transition rate $J$ can be correlated by the following relation

$$
G \propto b_{0} J
$$

Therefore, through eq $7,9,10,11$, and 12 , the growth 
rate $G$ is a function of the crystallization temperature, the crystal thickness, and the molecular weight. When the crystallization temperature and the molecular weight are held constant, $G(l)$ shows a maximum at the value of the thickness $l^{*}$ which satisfies the equation $\partial G / \partial l=0$. We define this thickness $l^{*}$ as the crystal thickness, since it is expected that the fast crystal growth is experimentally observed. ${ }^{22}$

A value of $\sigma_{\mathrm{eb}}=60 \mathrm{erg} \mathrm{cm}^{-2}$ was used in this calculation considering that it is necessary to hold the relation $\sigma_{\mathrm{eb}}<\sigma_{\mathrm{ef}}$ in the region where the bundlelike nucleation rate is more rapid than the foldedchain nucleation rate. Values of $b_{0}{ }^{2}=18 \times 10^{-16} \mathrm{~cm}^{2}$ and $c_{0}=1.27 \times 10^{-8} \mathrm{~cm}$ (from the study of polyethylene unit cell by Bunn ${ }^{23}$ ) and $\Delta h=2.8 \times 10^{9}$ $\mathrm{erg} \mathrm{cm}^{-3}$ (by Mandelkern et $a l^{24}$ ) were used. Selection of these values does not affect the qualitative results of this calculation. The estimated growth rates $G\left(l^{*}\right)$ are shown against the temperature variable $T_{\mathrm{m}}{ }^{0} / T \Delta T$ for three cases in Figure 7. It is clear that longer molecules give rise to higher growth rates. And, in general, $\left(\partial G\left(l^{*}\right) / \partial L\right)_{\Delta g}>0$ can be shown. The difference in growth rates, however, becomes small as the molecular weight is increased. This is similar to the results in Figure 4: the transition pressure depends little on molecular weight at a high molecular weight portion. At low supercoolings, the dependence of the growth rate on molecular weight becomes notable. For example, at $T_{\mathrm{m}}{ }^{0} / T \Delta T=10$, the ratio of the growth rate for $L=10000$ to that for $L=1000$ is about 20 ; however, at $T_{\mathrm{m}}{ }^{0} / T \Delta T=16$, the

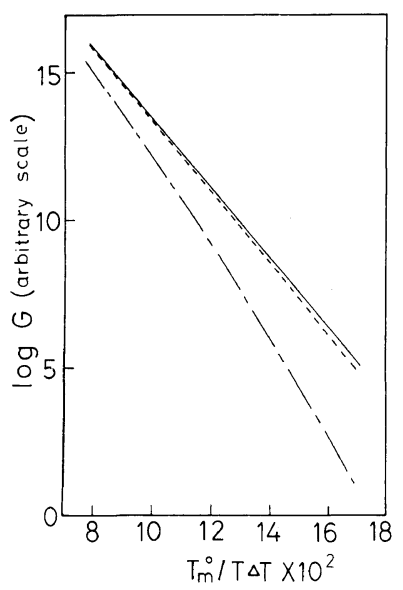

Figure 7. Plots of $\log G$ vs. $T_{\mathrm{m}}{ }^{0} / T \Delta T$ for (- - ) $L=1000$, (-----) $L=10000$, and (-) $L=100000$. Calculated for $\sigma=10 \mathrm{erg} \mathrm{cm}^{-2}$ and $\sigma_{\mathrm{eb}}=60 \mathrm{erg} \mathrm{cm}^{-2}$. ratio is about 4000 . Hence, at high pressure where the crystallization at low supercoolings was possible, crystallization for high molecular weight is considered favorable.

In the second nucleation stage, we consider a situation in which the remaining amorphous portions of the molecule begin to grow in the chain direction. This is schematically shown in Figure 8. At first, nucleation with the nucleus thickneess $l_{1}$ takes place in the chain direction and after completion of the lateral deposition, the next nucleation with the thickness $l_{2}$ takes place, and this process is repeated over and over. For these nucleation processes, we use the same surface nucleation rate equation as the first stage.

By using eq 8 , the transition rates were chosen in the following way.

$$
\begin{aligned}
a_{0 j}= & \frac{k T}{h} \exp \left[-\left\{2 b_{0} l_{j} \sigma\right.\right. \\
& +k T\left(\frac{n_{j}}{L}-\ln \frac{L_{j}-n_{j}}{L_{j}}\right) \\
& \left.\left.-0.5 b_{0}^{2} l_{j} \Delta g\right\} / k T\right] \\
\alpha_{1 j}= & \frac{k T}{h} \exp \left[-\left\{k T\left(\frac{n_{j}}{L}-\ln \frac{L_{j}-n_{j}}{L_{j}}\right)\right.\right. \\
& \left.\left.-0.5 b_{0}^{2} l_{j} \Delta g\right\} / k T\right]
\end{aligned}
$$

$$
\beta_{1 j}=\beta_{2 j}=\frac{k T}{h} \exp \left(-0.5 b_{0}^{2} l_{j} \Delta g / k T\right)
$$

where $L_{j}=L-\sum_{k=0}^{j-1} n_{k}+1$ and $n_{j}=l_{j} / c_{0}, j=1,2, \cdots$, and $n_{0}=l^{*} / c_{0}$ is set. Subscript $j$ denotes the $j$ th nucleation process.

The growth rate for $j$ th process can be expressed as

$$
u_{j} \propto l_{j} J_{j}
$$

where $J_{j}$ is the net transition rate for $j$ th nucleation process. We find the growth rate with the thickness which satisfies the equation $\partial u_{j} / \partial l_{j}=0$. Excep for the case of the low value of $L_{j}$, the longitudinal growth rates $u$ depended little on the number of nucleation processes, and these values are plotted in Figure 9. The dependence of $u$ on molecular weight is the same 


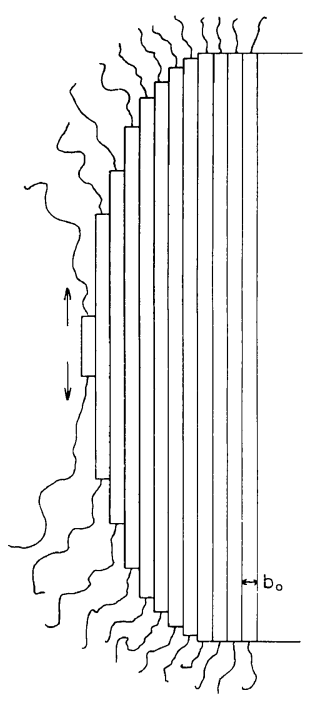

Figure 8. Model of the longitudinal growth of bundlelike nucleus seen from lateral direction. The arrows indicate the direction of longitudinal growth at a velocity $u$.

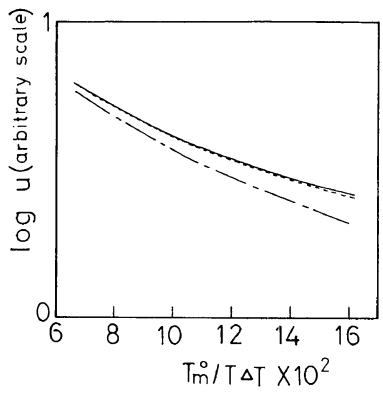

Figure 9. Plots of $\log u$ vs. $T_{\mathrm{m}}{ }^{0} / T \Delta T$ for (-$L=1000,(----) \quad L=10000$, and (-) $L=100000$. Calculated for $\sigma=10 \mathrm{erg} \mathrm{cm}^{-2}$.

as in the case of the first nucleation stage.

In actuality, it is considered for a bundle-like nucleus that further longitudinal growth is hindered by such factors as chain entaglement and deformations as crystallization proceeds. But such effects are not taken into account in this paper.

From the results of the first and second nucleation stages, it may be concluded that a higher-molecularweight sample is favorable for producing extendedchain crystals. Finally we mention that, for the crystallization from solution, the formation of bundle-like crystals is unfavorable mainly because of the concentration factor in the growth rate equation rendering the crystal growth considerably retarded.
Therefore, the formation of bundle-like crystals becomes difficult as the solvent concentration becomes higher. Indeed, it has been reported that for higher solvent concentrations, it becomes more difficult to produce extended-chain crystals. ${ }^{25,26}$

Acknowledgement. We wish to thank Dr. T. Saito of the Research Laboratories of Showa-Denko Company for supplying the fractionated samples.

\section{REFERENCES}

1. F. R. Anderson, J. Appl. Phys., 35, 64 (1964).

2. K. Kato, T. Nose, and T. Hata, Rep. Prog. Polym. Phys. Jpn., 14, 187 (1971).

3. T. Hatakeyama, T. Hashimoto, T. Ishida, M. Ohkuma, M. Kyotani, and H. Kanetsuna, Rep. Prog. Polym. Phys. Jpn., 14, 199 (1971).

4. D. C. Bassett and B. Turner, Phil. Mag., 29, 285 (1974).

5. S. Sawada and T. Nose, Polym. J., 11, 227 (1979).

6. D. C. Bassett and B. Turner, Nature (Phys. Sci), 240, 146 (1972).

7. S. Sawada and T. Nose, Polym. J., 11, 477 (1979).

8. T. Davidson and B. Wunderlich, J. Polym. Sci, A-2, 7, 377 (1969).

9. N. Yasuniwa, C. Nakafuku, and T. Takemura, Polym. J., 4, 526 (1973).

10. M. Kyotani and H. Kanetsuna, J. Polym. Sci., A-2, 12, 2331 (1974).

11. P. D. Calvert and D. R. Uhlmann, J. Polym. Sci., 10, 1811 (1972).

12. H. Kanetsuna, S. Mitsuhashi, M. Iguchi, T. Hatakeyama, M. Kyotani, and Y. Maeda, J. Polym. Sci., C, 42, 783 (1973).

13. J. D. Hoffman and J. I. Lauritzen, J. Res. Nat. Bur. Stand., A, 65, 297 (1961).

14. H. G. Zachmann, Kolloid Z. Z. Polym., 216-217, 180 (1967).

15. T. Hatakeyama, H. Kanetsuna, H. Kaneda, T. Hashimoto, J. Macromol. Sci., B 10, 359 (1974).

16. F. C. Frank and M. Tosi, Proc. R. Soc., London, Ser. A, 263, 323 (1961).

17. I. C. Sanchez and E. A. Dimarzio, J. Chem. Phys., 55, 893 (1971).

18. J. I. Lauritzen and J. D. Hoffman, J. Appl. Phys., 44, 4340 (1973).

19. P. J. Flory, J. Chem. Phys., 17, 223 (1949).

20. L. Mandelkern, J. Appl. Phys., 26, 443 (1955).

21. F. P. Price, "Nucleation", A. C. Zettlemoyer, Ed., Marcel-Dekker, New York, N.Y., 1969.

22. I. C. Sanchez and E. A. Dimarzio, Macromolecules, 4, 677 (1971).

23. C. W. Bunn, Trans. Faraday Soc., 35, 482 (1939).

24. L. Mandelkern, J. G. Fatou, and C. Howard, J. Phys. Chem., 68, 3386 (1964). 
S. SAwada, K. Kato, and T. Nose

25. G. Treiber, L. Melillo, and B. Wunderlich, J. Polym. 26. T. Arikawa, S. Miyata, and K. Sakaoku, Rep. Prog. Sci., Polym. Lett. Ed., 11, 435 (1973).

Polym. Phys. Jpn., 17, 229 (1974). 